\title{
On the number of ovals of a symmetry of a compact Riemann surface
}

\author{
Emilio Bujalance, Francisco Javier Cirre, José Manuel Gamboa \\ and Grzegorz Gromadzki
}

\begin{abstract}
Let $X$ be a symmetric compact Riemann surface whose full group of conformal automorphisms is cyclic. We derive a formula for counting the number of ovals of the symmetries of $X$ in terms of few data of the monodromy of the covering $X \rightarrow X / G$, where $G=\mathrm{Aut}^{ \pm} X$ is the full group of conformal and anticonformal automorphisms of $X$.
\end{abstract}

\section{Introduction}

A symmetry on a compact Riemann surface $X$ is an anticonformal involution $\tau: X \rightarrow X$, and surfaces admitting some symmetry are called symmetric. Under the well known equivalence between compact Riemann surfaces and (smooth, projective) complex algebraic curves, the symmetries correspond to real algebraic curves, that is, curves which may be defined over the field of real numbers. The fixed point set of a symmetry is either empty or consists of a disjoint union of simple closed curves, called ovals in Hilbert's terminology. These correspond to the connected components of the set of $\mathbb{R}$-rational points of the associated real algebraic curve.

The study of ovals on surfaces is a classical problem started at the end of the XIX century by Harnack [6], Klein [7] and Weichold [15], among others. Concerning the computation of the number of ovals, the seminal result is Harnack's bound, which states that the maximum number of ovals of a symmetry on a genus $g$ compact Riemann surface is $g+1$. More recently, efforts have been directed to obtain bounds for the sum of the number of ovals of all symmetries of a surface of genus $g$. Natanzon [12] showed, among other things, that this sum does not exceed $42(g-1)$, and in [4] this bound 
was improved to $12(g-1)$ (for $g \neq 2,3,5,7,9)$ and proved to be sharp for infinitely many values of $g$. Other bounds for the sum of the number of ovals of $k$ non-conjugate symmetries are due to Singerman [14] and the fourth author [5]. Related results on ovals of symmetries appear in $[9,11]$.

On the other hand, there are few results concerning the difficult task of computing the precise value of the number of ovals of symmetries. This paper deals with this problem in the case of Riemann surfaces $X$ whose full group $\mathrm{Aut}^{+} X$ of conformal automorphisms is cyclic (although our results are valid in a more general context, see Remark 2.6). Let $\operatorname{Aut}^{ \pm} X$ be the full group of conformal and anticonformal automorphisms of $X$. We derive a formula in terms of surprisingly few data of the monodromy of the covering $X \rightarrow X /$ Aut $^{ \pm} X$ (see Theorem 2.1) which provides the precise value of the sum of the number of ovals of the conjugacy classes of symmetries of $X$. The main result in [2] can be seen as a particular case of this formula, see also [8]. In Section 3 we obtain sharp bounds for this sum in terms of the genus $g$ and of the nature of the 2-extension $\mathrm{Aut}^{ \pm} X$ of the cyclic group $\mathrm{Aut}^{+} X$. Some applications to compute the number of ovals of a single symmetry are given in Section 2. In the proofs we use the combinatorial theory of Riemann surfaces and non-euclidean crystallographic groups.

\section{Preliminaries}

A non-Euclidean crystallographic (NEC) group is a discrete group of (orientation preserving or reversing) isometries of the hyperbolic plane $U$ with compact quotient space. The signature $\sigma(\Lambda)$ of an NEC group $\Lambda$ is a collection of non-negative integers and symbols of the form

$$
\sigma(\Lambda)=\left(h ; \pm ;\left[m_{1}, \ldots, m_{r}\right] ;\left\{\left(n_{11}, \ldots, n_{1 s_{1}}\right), \ldots,\left(n_{k 1}, \ldots, n_{k s_{k}}\right)\right\}\right)
$$

which determines the algebraic structure of $\Lambda$ and the geometric structure of its quotient orbifold $U / \Lambda$. This has genus $h, k \geq 0$ boundary components, and it is orientable if the sign "+" occurs, and non-orientable otherwise. The integers $m_{1}, \ldots, m_{r}$ are the proper periods of $\Lambda$, and represent the branching over interior points of $U / \Lambda$ in the natural projection $U \rightarrow U / \Lambda$. The $k$ brackets $\left(n_{i 1}, \ldots, n_{i s_{i}}\right)$ are the period cycles and represent the branching over the $i$-th boundary component. The integers $n_{i j}$ are the link periods. An empty period cycle corresponds to a boundary component with no ramification over it, i.e., it has no link periods, and it will be represented by (-). For short, we say that a period cycle of $\Lambda$ is odd if it is non-empty and all its link periods are odd. The signature also determines the algebraic structure of $\Lambda$. It has generators 
- $x_{1}, \ldots, x_{r}$ (elliptic isometries)

- $c_{10}, \ldots, c_{1 s_{1}}, \ldots, c_{k 0}, \ldots, c_{k s_{k}}$ (reflections)

- $e_{1}, \ldots, e_{k}$ (orientation preserving isometries, which will be called connecting generators)

- $a_{1}, b_{1}, \ldots, a_{h}, b_{h}$ (hyperbolic isometries) if the sign of $\sigma(\Lambda)$ is " + " or $d_{1}, \ldots, d_{h}$ (glide reflections) otherwise.

and relations

- $x_{i}^{m_{i}}=1$ for $i=1, \ldots, r$;

- $c_{i, j-1}^{2}=c_{i j}^{2}=\left(c_{i, j-1} c_{i j}\right)^{n_{i j}}=1$, for $i=1, \ldots, k$, and $j=1, \ldots, s_{i}$;

- $e_{i} c_{i 0} e_{i}^{-1} c_{i s_{i}}=1$

- $x_{1} \cdots x_{r} e_{1} \cdots e_{k}\left[a_{1}, b_{1}\right] \cdots\left[a_{h}, b_{h}\right]=1$ if the sign "+" occurs, and $x_{1} \cdots x_{r} e_{1} \cdots e_{k} d_{1}^{2} \cdots d_{h}^{2}$ otherwise.

A set of generators as the above is called a set of canonical generators. Any reflection in $\Lambda$ is conjugate within $\Lambda$ to a canonical one. Moreover, it follows from the presentation that all the canonical reflections of an odd period cycle are mutually conjugate, and that the number of conjugacy classes of reflections of a period cycle with $v>0$ even link periods is $v$.

The area of a fundamental domain for an NEC group $\Lambda$ with signature (1.1) is

$$
\mu(\Lambda)=2 \pi\left(\eta h+k-2+\sum_{i=1}^{r}\left(1-\frac{1}{m_{i}}\right)+\frac{1}{2} \sum_{i=1}^{k} \sum_{j=1}^{s_{i}}\left(1-\frac{1}{n_{i j}}\right)\right),
$$

where $\eta=1$ if the sign "-" occurs and $\eta=2$ otherwise. If $\Delta$ is a subgroup of $\Lambda$ of finite index then the Riemann-Hurwitz formula reads

$$
\mu(\Delta)=[\Lambda: \Delta] \mu(\Lambda) .
$$

For each compact Riemann surface $X$ of genus $g \geq 2$ there exists a surface Fuchsian group $\Gamma$ i.e., a torsion free NEC group with sign "+", such that $X$ is isomorphic to the quotient surface $U / \Gamma$. Each subgroup $G$ of the full group $\mathrm{Aut}^{ \pm} X$ of conformal and anticonformal automorphisms of $X$ is isomorphic to a quotient $\Lambda / \Gamma$ for some NEC group $\Lambda$ containing $\Gamma$ as a normal subgroup. Hence, there exists an epimorphism $\theta: \Lambda \rightarrow G$ with $\operatorname{ker} \theta=\Gamma$. Epimorphisms as the above whose kernel is a surface Fuchsian group will be called smooth. The subgroup of Aut ${ }^{ \pm} X$ consisting of conformal automorphisms will be denoted by $\mathrm{Aut}^{+} X$. 
Let $\tau$ be a symmetry on a compact Riemann surface $X$ of genus $g \geq 2$, and let $u$ be a conformal automorphism of $X$ of order $n$ such that $\tau$ normalizes $\langle u\rangle$, that is, $\tau u \tau \in\langle u\rangle$. Then the group $\langle u, \tau\rangle$ has a presentation of the form

$$
G_{n}^{\alpha}:=\left\langle u, \tau \mid u^{n}=\tau^{2}=1, \tau u \tau=u^{\alpha}\right\rangle,
$$

where $\alpha^{2} \equiv 1(\bmod n)$. For example, $G_{n}^{\alpha}$ is abelian if and only if $\alpha=1$, and dihedral if and only if $\alpha=n-1$.

Notice that if $\mathrm{Aut}^{+} X=\langle u\rangle$ is cyclic then $\tau$ normalizes $\langle u\rangle$, and so $\mathrm{Aut}^{ \pm} X=G_{n}^{\alpha}$ for some $\alpha$.

Viewing $G_{n}^{\alpha}$ as a group of automorphisms of $X$, those involutions in $G_{n}^{\alpha}$ which can be written with an odd number of occurrences of the letter $\tau$ are symmetries of $X$, and so we call them the symmetries of $G_{n}^{\alpha}$. We will represent by $\|s\|$ the number of ovals of a symmetry $s$.

We fix the following notations. The cyclic group of order $n$ will be denoted by $C_{n}$. We set

$$
d=\operatorname{gcd}(n, \alpha+1) \quad \text { and } \quad f=\operatorname{gcd}(n, \alpha-1),
$$

where gcd stands for the greatest common divisor. Note that $\operatorname{gcd}(d, f)=1$ if $n$ is odd and $\operatorname{gcd}(d, f)=2$ otherwise.

Let us state first a purely group theoretic lemma concerning the conjugacy classes of symmetries in $G_{n}^{\alpha}$.

Lemma 1.1 A set of representatives of all the conjugacy classes of symmetries in $G_{n}^{\alpha}$ is the following:

- $\{\tau\} \quad$ if $n$ is odd or $\alpha^{2} \not \equiv 1(\bmod 2 n)$;

- $\left\{\tau, u^{n / d} \tau\right\} \quad$ if $n$ is even and $\alpha^{2} \equiv 1(\bmod 2 n)$.

Proof. It is easy to check that the symmetries in $G_{n}^{\alpha}$ are of the form $u^{j n / d} \tau$ for $j=1, \ldots, d$. Conjugation by $u$ gives $u^{j n / d} \tau \sim u^{(\alpha-1)+j n / d} \tau$; hence, conjugation by $u$ groups the $d$ symmetries into sets of $h:=\operatorname{ord}\left(u^{\alpha-1}\right)=n / f$ elements. We claim that $h=d$ or $d / 2$. First, $h \mid d$ because $h \mid n$ and also $h \mid(\alpha+1)$ since $\left(u^{\alpha-1}\right)^{\alpha+1}=u^{\alpha^{2}-1}=1$. Now, $d / h$ divides $\alpha+1$, and $d / h$ also divides $n / h=f$ which in turn divides $\alpha-1$; hence $d / h$ divides $\operatorname{gcd}(\alpha+1, \alpha-1)=1$ or 2 , proving our claim. If $h=d$ then the $d$ symmetries $u^{j n / d} \tau$ are all conjugate (via powers of $u$ ) to $\tau$, for instance. If, on the contrary, $d=2 h$ then all the symmetries $u^{j n / d} \tau$ are conjugate via powers of $u$ to either $\tau$ or $u^{n / d} \tau$. Since the other generator $\tau$ of $G_{n}^{\alpha}$ does not conjugate these two symmetries, they are representatives of the two conjugacy classes of symmetries in $G_{n}^{\alpha}$. Finally, observe that $d=2 h$ if and only if $\operatorname{gcd}(n, \alpha+1) \operatorname{gcd}(n, \alpha-1)=2 n$ and this happens if and only if $n$ is even and $2 n$ divides $(\alpha+1)(\alpha-1)$. 
Remark 1.2 We have just shown that $n / f=d$ or $n / f=d / 2$. In fact,

i) If $n$ is odd or $\alpha^{2} \not \equiv 1(\bmod 2 n)$ then $n=d f$ and all of the $d$ symmetries in $G_{n}^{\alpha}$ are conjugate to $\tau$.

ii) If $n$ is even and $\alpha^{2} \equiv 1(\bmod 2 n)$ then $2 n=d f$ and $d / 2$ symmetries in $G_{n}^{\alpha}$ are conjugate to $\tau$ and the other $d / 2$ to $u^{n / d} \tau$.

In order to compute the number of ovals of a symmetry we need a description of the centralizer of a reflection $c$ in an NEC group. Singerman [13] proved that this centralizer is $C_{2} \oplus C_{\infty}$, if $c$ corresponds to an empty or an odd period cycle, and to $C_{2} \oplus\left(C_{2} * C_{2}\right)$ otherwise, where $C_{\infty}$ is the cyclic group of infinite order, and $*$ stands for the free product. Going a bit more into the details of Singerman's proof, one can find explicitly the generators of this group.

Lemma 1.3 Let e, $c_{0}, \ldots, c_{s}$ be a set of canonical generators associated to a period cycle $\left(n_{1}, \ldots, n_{s}\right)$ of an $N E C$ group $\Lambda$ and let $\mathrm{C}\left(\Lambda, c_{i}\right)$ be the centralizer in $\Lambda$ of $c_{i}$.

(i) If $s=0$ then $\mathrm{C}\left(\Lambda, c_{0}\right)=\left\langle c_{0}\right\rangle \oplus\langle e\rangle$.

(ii) If $s \neq 0$ and all $n_{i}$ are odd then $\mathrm{C}\left(\Lambda, c_{0}\right)=\left\langle c_{0}\right\rangle \oplus\left\langle\prod_{i=0}^{s-1}\left(c_{i+1} c_{i}\right)^{\left(n_{i+1}-1\right) / 2} e\right\rangle$.

(iii) If $n_{i}, n_{j}$ are even and $n_{i+1}, \ldots, n_{j-1}$ are odd $(i<j \leq s)$ then

$$
\mathrm{C}\left(\Lambda, c_{i}\right)=\left\langle c_{i}\right\rangle \oplus\left(\left\langle\left(c_{i-1} c_{i}\right)^{n_{i} / 2}\right\rangle *\left\langle x^{-1}\left(c_{j-1} c_{j}\right)^{n_{j} / 2} x\right\rangle\right)
$$

where $x=\left(c_{j-2} c_{j-1}\right)^{\left(n_{j-1}-1\right) / 2} \cdots\left(c_{i} c_{i+1}\right)^{\left(n_{i+1}-1\right) / 2}$.

(iv) If $n_{i}$ is even, $n_{i+1}, \ldots, n_{s}, n_{1}, \ldots, n_{j-1}$ are odd and $n_{j}$ is even with $1 \leq j \leq i$ then

$$
\begin{array}{r}
\mathrm{C}\left(\Lambda, c_{i}\right)=\left\langle c_{i}\right\rangle \oplus\left(\left\langle\left(c_{i-1} c_{i}\right)^{n_{i} / 2}\right\rangle *\left\langle x^{-1}\left(c_{j-1} c_{j}\right)^{n_{j} / 2} x\right\rangle\right), \\
\text { where } x=\prod_{t=1}^{j-1}\left(c_{j-1-t} c_{j-t}\right)^{\left(n_{j-t}-1\right) / 2} e^{-1} \prod_{t=0}^{s-i-1}\left(c_{s-1-t} c_{s-t}\right)^{\left(n_{s-t}-1\right) / 2} .
\end{array}
$$

Proof. Claim $(i)$ is actually proved by Singerman in [13]. For $(i i)$, observe that $\beta=e^{-1}\left(c_{s-1} c_{s}\right)^{\left(n_{s}-1\right) / 2} \ldots\left(c_{1} c_{2}\right)^{\left(n_{2}-1\right) / 2}\left(c_{0} c_{1}\right)^{\left(n_{1}-1\right) / 2}$ belongs to $\mathrm{C}\left(\Lambda, c_{0}\right)$, which gives one of the inclusions. For the converse, let $F$ be a fundamental region for $\Lambda$, and let $\gamma_{0}, \gamma_{1}, \ldots, \gamma_{s}$ be the part of the surface symbol for $F$ corresponding to the period cycle $\left(n_{1}, \ldots n_{s}\right)$. The axis $\ell$ of $c_{0}$ splits into intervals being edges of the images of $F$ abuting $\ell$, each segment having 
a label from the surface symbol to which it belongs. Now for $\lambda \in \Lambda$, the element $\lambda c_{0} \lambda^{-1}$ is a reflection with axis $\lambda(\ell)$. So $\lambda$ centralizes $c_{0}$ if and only if $\lambda(\ell)=\ell$ while the last is true if and only if $\lambda(F)$ abuts $\ell$ at an edge labelled by $\gamma_{0}$. Thus there is a bijective correspondence between segments of $\ell$ labelled by $\gamma_{0}$ and elements of $\mathrm{C}\left(\Lambda, c_{0}\right)$. Finally

$$
\left(c_{i} c_{i+1}\right)^{\left(n_{i+1}-1\right) / 2} c_{i}\left(c_{i} c_{i+1}\right)^{-\left(n_{i+1}-1\right) / 2}=c_{i+1} \text { and } e^{-1} c_{s} e=c_{0} .
$$

So the segment labelled by $\gamma_{i}$ is followed by $\gamma_{i+1}$ for $i=0, \ldots, s-1$ while $\gamma_{s}$ by $\gamma_{0}$. Therefore $\gamma_{0}, \gamma_{1}, \ldots, \gamma_{s}, \gamma_{0}$ are the labels of consecutive segments on $\ell$ and this labelling repeats on $\ell$ periodically. Hence $c_{0}$ and $\beta$ generate $\mathrm{C}\left(\Lambda, c_{0}\right)$ indeed.

Proofs of claims (iii) and (iv) are similar and we omit them.

\section{Main result}

Let $X=U / \Gamma$ be a symmetric Riemann surface whose full group of conformal automorphisms $\mathrm{Aut}^{+} X$ is cyclic of order $n$, say $\operatorname{Aut}^{+} X=\langle u\rangle$. Then $\mathrm{Aut}^{ \pm} X=G_{n}^{\alpha}$ for some $\alpha$, where $G_{n}^{\alpha}$ has presentation (1.4). Let $\theta: \Lambda \rightarrow G_{n}^{\alpha}=\langle u, \tau\rangle$ be the associated smooth epimorphism with $\operatorname{ker} \theta=\Gamma$. Let us write $\theta\left(e_{i}\right)=u^{\varepsilon_{i}}$ where $e_{1}, \ldots, e_{k}$ is a set of connecting generators associated to the $k$ period cycles of $\Lambda$. Recall that a non-empty period cycle is said to be odd if all its link periods are odd. The next theorem shows the close relation between the integers $\varepsilon_{i}$ and the number of ovals of the symmetries of $X$. We stress that this result is stated for surfaces with $\mathrm{Aut}^{+} X$ cyclic, but it can be applied to a wider variety of Riemann surfaces, see Remark 2.6.

Theorem 2.1 With the above notations, let us consider the following subsets of $\{1, \ldots, k\}$ :

- $T=\left\{i \mid\right.$ the $i^{\text {th }}$ period cycle of $\Lambda$ is empty $\}$;

- $S_{1}=\left\{i \mid\right.$ the $i^{\text {th }}$ period cycle of $\Lambda$ is odd and $\frac{\varepsilon_{i}(\alpha+1)}{\operatorname{gcd}\left(\varepsilon_{i}(\alpha+1), n\right)}$ is odd $\}$;

- $S_{2}=\left\{i \mid\right.$ the $i^{\text {th }}$ period cycle of $\Lambda$ is odd and $\frac{\varepsilon_{i}(\alpha+1)}{\operatorname{gcd}\left(\varepsilon_{i}(\alpha+1), n\right)}$ is even $\}$.

Let $v$ be the total number of even link periods in the signature of $\Lambda$. Then

$$
\begin{gathered}
\|\tau\|+\left\|u^{n / d} \tau\right\|=\sum_{i \in T} \frac{2 \operatorname{gcd}\left(\varepsilon_{i}, n\right)}{d}+\sum_{i \in S_{1}} \operatorname{gcd}\left(\frac{n}{d}, \varepsilon_{i}\right) \\
+2 \sum_{i \in S_{2}} \operatorname{gcd}\left(\frac{n}{d}, \varepsilon_{i}\right)+v \frac{n}{d} .
\end{gathered}
$$

If $\Lambda$ has no period cycle then $\|\tau\|=0$ for each symmetry $\tau$ in $X$. 
Proof . Observe first that each $\theta\left(e_{i}\right)$ is indeed of the form $u^{\varepsilon_{i}}$ since $\theta\left(e_{i}\right)$ belongs to $\langle u\rangle$, the orientation preserving subgroup of $G_{n}^{\alpha}$. If $\Lambda$ has no period cycle then $\Lambda$ contains no reflection and each symmetry $\tau$ in $G_{n}^{\alpha}$ is the image of a glide reflection. It follows that the fixed point set of $\tau$ is empty. If $\Lambda$ has some period cycle then the image $\theta(c)$ of each canonical reflection $c$ is a symmetry $s$ whose fixed point set is non-empty. In this case, Theorem 3.1 in [4] shows that $\|s\|=\sum_{i}\left[\mathrm{C}\left(G_{n}^{\alpha}, \theta\left(c_{i}\right)\right): \theta\left(\mathrm{C}\left(\Lambda, c_{i}\right)\right)\right]$ where $\mathrm{C}(G, g)$ denotes the centralizer in the group $G$ of the element $g \in G$ and the sum is taken over a set of representatives of conjugacy classes of canonical reflections in $\Lambda$ whose images under $\theta$ are conjugate within $G_{n}^{\alpha}$ to $s$.

Assume first that $n$ is even and $\alpha^{2} \equiv 1(\bmod 2 n)$. Then each $\theta\left(c_{i}\right)$ is conjugate to either $\tau$ or $u^{n / d} \tau$, and so

$$
\|\tau\|+\left\|u^{n / d} \tau\right\|=\sum_{i}\left[\mathrm{C}\left(G_{n}^{\alpha}, \theta\left(c_{i}\right)\right): \theta\left(\mathrm{C}\left(\Lambda, c_{i}\right)\right)\right]
$$

where now the sum is taken over all representatives of conjugacy classes of canonical reflections in $\Lambda$. There are $t+s+v$ of such classes, where $t$ is the number of empty period cycles, $s$ is the number of odd period cycles, and $v$ is the total number of even link periods in the signature of $\Lambda$. So the sum in (2.2) has terms of three types.

For those terms corresponding to reflections $c_{i}$ associated to empty period cycles, part (i) in Lemma 1.3 yields $\left|\theta\left(\mathrm{C}\left(\Lambda, c_{i}\right)\right)\right|=2 \operatorname{ord}\left(u^{\varepsilon_{i}}\right)=2 n / \operatorname{gcd}\left(\varepsilon_{i}, n\right)$.

Let $c_{i}$ be a reflection such that the corresponding link period $n_{i}$ is even. Parts (iii) and (iv) in Lemma 1.3 show that $\left|\theta\left(\mathrm{C}\left(\Lambda, c_{i}\right)\right)\right|=2|H|$ where $H$ is the image of the group $\left\langle\left(c_{i-1} c_{i}\right)^{n_{i} / 2}\right\rangle *\left\langle x^{-1}\left(c_{j-1} c_{j}\right)^{n_{j} / 2} x\right\rangle$ for a certain $x \in \Lambda$. In fact, $x$ is an orientation preserving element and so the image of $H$ lies in the cyclic group $\langle u\rangle$; in addition, $\theta\left(c_{i-1} c_{i}\right)^{n_{i} / 2}=\theta\left(c_{j-1} c_{j}\right)^{n_{j} / 2}=u^{n / 2}$. It follows that $\left|\theta\left(\mathrm{C}\left(\Lambda, c_{i}\right)\right)\right|=4$.

Let us consider now an odd period cycle $\left(n_{1}, \ldots, n_{s}\right)$ of $\Lambda$. Let $c_{0}, \ldots, c_{s}$ be a set of canonical reflections associated to it, and $e$ be its connecting generator, with image $\theta(e)=u^{\varepsilon}$. The image $\theta\left(c_{i}\right)$ of each reflection $c_{i}$ is a symmetry in $G_{n}^{\alpha}=\langle u, \tau\rangle$ and so it is of the form $\theta\left(c_{i}\right)=u^{h_{i} n / d} \tau$ for some $h_{i} \in\{1, \ldots, d\}$. In particular,

$$
\theta\left(c_{i+1} c_{i}\right)=u^{\left(h_{i+1}+\alpha h_{i}\right) n / d}=u^{\left(h_{i+1}-h_{i}\right) n / d},
$$

and from the relation $e c_{0}=c_{s} e$ we get $\varepsilon(\alpha-1) \equiv\left(h_{0}-h_{s}\right) n / d(\bmod n)$. Part (ii) in Lemma 1.3 shows that the order of $\theta\left(\mathrm{C}\left(\Lambda, c_{0}\right)\right)$ is twice the order of $\prod_{i=0}^{s-1} \theta\left(c_{i+1} c_{i}\right)^{\left(n_{i+1}-1\right) / 2} \theta(e)$, which is an element of the form $u^{h}$. It is easier to compute the order of its square $u^{2 h}$. Indeed, $\theta\left(c_{i+1} c_{i}\right)^{n_{i+1}-1}=$ $\theta\left(c_{i+1} c_{i}\right)^{-1}=u^{\left(h_{i}-h_{i+1}\right) n / d}$ and so

$$
2 h \equiv \sum_{i=0}^{s-1}\left(h_{i}-h_{i+1}\right) n / d+2 \varepsilon \equiv\left(h_{0}-h_{s}\right) n / d+2 \varepsilon \equiv \varepsilon(\alpha+1) \quad(\bmod n) .
$$


Comparing $\operatorname{gcd}(n, 2 h)$ with $\operatorname{gcd}(n, h)$ yields that

$$
\operatorname{ord}\left(u^{h}\right)= \begin{cases}\frac{n}{\operatorname{gcd}(\varepsilon(\alpha+1), n)} & \text { if } \frac{\varepsilon(\alpha+1)}{\operatorname{gcd}(\varepsilon(\alpha+1), n)} \quad \text { is even, } \\ \frac{2 n}{\operatorname{gcd}(\varepsilon(\alpha+1), n)} & \text { otherwise. }\end{cases}
$$

Since $(\alpha+1) / d$ and $n / d$ are coprime, we may replace $\operatorname{gcd}(\varepsilon(\alpha+1), n)$ by $\operatorname{gcd}(\varepsilon d, n)$. Therefore

$$
\left|\theta\left(\mathrm{C}\left(\Lambda, c_{0}\right)\right)\right|= \begin{cases}\frac{2 n}{\operatorname{gcd}(\varepsilon d, n)} & \text { if } \frac{\varepsilon(\alpha+1)}{\operatorname{gcd}(\varepsilon(\alpha+1), n)} \text { is even } \\ \frac{4 n}{\operatorname{gcd}(\varepsilon d, n)} & \text { otherwise. }\end{cases}
$$

Finally, since $d / 2$ of the $d$ symmetries of $G_{n}^{\alpha}$ are conjugate to $\theta\left(c_{0}\right)$ we get $\left|\mathrm{C}\left(G_{n}^{\alpha}, \theta\left(c_{0}\right)\right)\right|=\left|G_{n}^{\alpha}\right| /(d / 2)=4 n / d$. Formula (2.1) follows easily.

In case $n$ is odd or $\alpha^{2} \not \equiv 1(\bmod 2 n)$, all the images of the canonical reflections in $\Lambda$ are conjugate to $\tau$. So the sum in the right hand side in (2.2) gives just $\|\tau\|$; but in this case $\left|\mathrm{C}\left(G_{n}^{\alpha}, \theta\left(c_{i}\right)\right)\right|=\left|G_{n}^{\alpha}\right| / d=2 n / d$, which is half of the value obtained above. On the other hand, the value of $\left|\theta\left(\mathrm{C}\left(\Lambda, c_{i}\right)\right)\right|$ is the same as in the preceding case, and so

$$
\|\tau\|=\sum_{i \in T} \frac{\operatorname{gcd}\left(\varepsilon_{i}, n\right)}{d}+\sum_{i \in S_{1}} \frac{1}{2} \operatorname{gcd}\left(\frac{n}{d}, \varepsilon_{i}\right)+\sum_{i \in S_{2}} \operatorname{gcd}\left(\frac{n}{d}, \varepsilon_{i}\right)+v \frac{n}{2 d} .
$$

Therefore $\|\tau\|+\left\|u^{n / d} \tau\right\|=2\|\tau\|$ coincides with the right hand side in (2.1).

Remark 2.2 Note that in order to count the number of ovals of symmetries of $X$ we do not require the complete knowledge of the monodromy of the covering $X \rightarrow X / G_{n}^{\alpha}$. In fact, Formula (2.1) just involves the number of odd and empty period cycles, the number of even link periods and the images under $\theta$ of the connecting generators of $\Lambda$.

Formula (2.1) can be written in a much simpler way in case $n$ is odd.

Corollary 2.3 With the above notations, if $n$ is odd then

$$
\|\tau\|=\sum_{i=1}^{k} \operatorname{gcd}\left(\varepsilon_{i}, f\right) .
$$

Proof. If $n$ is odd then $n / d=f$ and $v=0$. So, the $k$ period cycles of $\Lambda$ are either empty or odd. Moreover, $S_{1}$ is empty since $\varepsilon_{i}(\alpha+1)=2 h$ is even for each $\varepsilon_{i}$ (see the proof of Theorem 2.1) and $\operatorname{gcd}\left(\varepsilon_{i}(\alpha+1), n\right)$ is odd. Now, for each $i \in T$, we have $\operatorname{gcd}\left(\varepsilon_{i}, n\right) / d=\operatorname{gcd}\left(\varepsilon_{i} / d, f\right)=\operatorname{gcd}\left(\varepsilon_{i}, f\right)$, where in the last equality we have used that $d$ and $f$ are coprime. 
Remarks 2.4 (1) As particular cases of Corollary 2.3, if $\alpha=n-1\left(G_{n}^{\alpha}\right.$ dihedral) then $f=1$ and so $\|\tau\|=k$, which coincides with [2, Theorem 2(ii)]; if $\alpha=1\left(G_{n}^{\alpha}\right.$ cyclic) then $f=n$ and so $\|\tau\|=\sum_{i=1}^{k} \operatorname{gcd}\left(\varepsilon_{i}, n\right)=$ $\sum_{i=1}^{k} n / \operatorname{ord}\left(\theta\left(e_{i}\right)\right)$, which coincides with [10, Theorem 1(e)].

(2) If $n$ is even and $\alpha^{2} \equiv 1(\bmod 2 n)$ then $\tau$ and $u^{n / d} \tau$ are not conjugate. Formula (2.1) provides the sum of the number of ovals of both symmetries, but in this case, it does not give us the precise value of each summand. In fact, the values of $\|\tau\|$ and $\left\|u^{n / d} \tau\right\|$ depend not only on the images $\theta\left(e_{i}\right)$ but also on the images of each canonical reflection. Indeed, it follows from the proof of Theorem 2.1 that

$$
\|\tau\|=\sum_{i} \frac{2 \operatorname{gcd}\left(\varepsilon_{i}, n\right)}{d}+\sum_{i} \operatorname{gcd}\left(\frac{n}{d}, \varepsilon_{i}\right)+2 \sum_{i} \operatorname{gcd}\left(\frac{n}{d}, \varepsilon_{i}\right)+v_{\tau} \frac{n}{d}
$$

where the sums restrict to those period cycles (empty or odd) for which the images of the associated canonical reflections are conjugate to $\tau$, and $v_{\tau}$ is the number of even link periods $n_{i j}$ in $\sigma(\Lambda)$ such that the image $\theta\left(c_{i j}\right)$ of the corresponding canonical reflection is conjugate to $\tau$. An analogous formula holds for $\left\|u^{n / d} \tau\right\|$.

(3) If $n$ is even and $G_{n}^{\alpha}$ is dihedral (that is, $d=n$ ) then

$$
\|\tau\|+\|u \tau\|=\sum 2 \operatorname{gcd}\left(\varepsilon_{i}, n\right) / d+\operatorname{card}\left(S_{1}\right)+2 \operatorname{card}\left(S_{2}\right)+v .
$$

This is the value which should be given in Theorem 2(i) in [2] to make it correct. This mistake was pointed out in [8], where upper and lower bounds for $\|\tau\|+\|u \tau\|$ are given.

Example 2.5 Let us consider compact symmetric Riemann surfaces of genus $g=5$ on which the cyclic group $C_{8}$ acts as a group of conformal automorphisms. Let $\tau$ be a symmetry on such a surface $X$. We claim that:

1. If $C_{8}=$ Aut $^{+} X$ then $\|\tau\| \leq 3$. Conversely, for each $k \leq 3$ there exist a surface $X_{k}$ of genus 5 such that $C_{8}=$ Aut $^{+} X_{k}$, and a symmetry $\tau_{k}$ on $X_{k}$ with $\left\|\tau_{k}\right\|=k$.

2. If $C_{8} \subset$ Aut $^{+} X$ and $\tau$ normalizes $C_{8}$, then $\|\tau\| \leq 5$. Conversely, for each $k \leq 5$ there exist a surface $X_{k}$ of genus 5 such that $C_{8} \subset$ Aut $^{+} X_{k}$, and a symmetry $\tau_{k}$ on $X_{k}$ with $\left\|\tau_{k}\right\|=k$.

In fact, if $C_{8}$ acts on a genus 5 surface $X=U / \Gamma$ then $C_{8}=\Delta / \Gamma$ for some Fuchsian group $\Delta$ whose signature (by Riemann-Hurwitz formula) is either $\sigma_{1}=(0 ;+;[2,4,8,8] ;\{-\})$ or $\sigma_{2}=(1 ;+;[2,2] ;\{-\})$. Theorem 4.1 in [1] on the extendability of cyclic group actions yields that if $\sigma(\Delta)=\sigma_{2}$ 
then $\mathrm{Aut}^{+} X$ is strictly larger than $C_{8}$. Let $\tau$ be a symmetry on $X$ which normalizes $C_{8}=\langle u\rangle$. Then Aut ${ }^{ \pm} X$ contains $\langle u, \tau\rangle=G_{8}^{\alpha}$ for some $\alpha$ and so there exist an NEC group $\Lambda$ and a smooth epimorphism $\theta: \Lambda \rightarrow G_{8}^{\alpha}$ with $\operatorname{ker} \theta=\Gamma$. Observe that the canonical Fuchsian subgroup $\Lambda^{+}$of $\Lambda$ equals $\Delta$. Using Corollary 2.2.5 in [3] we get that there exist two possible signatures for $\Lambda$ if $\sigma(\Delta)=\sigma_{1}$ and six if $\sigma(\Delta)=\sigma_{2}$. The "if" parts of statements 1 and 2 follow easily from formula (2.5) and a detailed analysis of the possible epimorphisms $\theta: \Lambda \rightarrow G_{8}^{\alpha}$. For the converse parts, we also have to take into account that $\sigma_{1}$ is a maximal Fuchsian signature (see [3, Section 5.1] for the definition of maximal signature). So if $\sigma(\Delta)=\sigma_{1}$ then $X$ can be chosen such that $\mathrm{Aut}^{+} X=C_{8}$.

The largest values (4 and 5) for the number of ovals of a symmetry, can be attained by two symmetries of the same surface. Indeed, let us consider an NEC group $\Lambda$ with signature $(0 ;+;[-] ;\{(2,2),(-)\})$ and the smooth epimorphism $\theta: \Lambda \rightarrow G_{8}^{1}=\langle u\rangle \times\langle\tau\rangle$ given by

$$
c_{10} \mapsto u^{4} \tau, \quad c_{11} \mapsto \tau, \quad c_{12} \mapsto u^{4} \tau, \quad e_{1} \mapsto u, \quad c_{20} \mapsto \tau, \quad e_{2} \mapsto u^{-1}
$$

Then $X=U / \operatorname{ker} \theta$ is a genus 5 Riemann surface which admits two symmetries $\tau$ and $u^{4} \tau$ such that $\|\tau\|=5$ and $\left\|u^{4} \tau\right\|=4$, as formula (2.5) easily shows.

Remark 2.6 Theorem 2.1 may be seen as an approach to the solution of the problem of counting the number of ovals of a symmetry $\tau$ on a compact Riemann surface $X$. Although the theorem is stated for surfaces with cyclic full conformal automorphism group, it can also be applied to a wider variety of Riemann surfaces. Let $\tau$ be a symmetry of an arbitrary Riemann surface $X$ which admits a non-trivial conformal automorphism $u$ such that $\tau$ normalizes $\langle u\rangle$. Then $\langle u, \tau\rangle$ is of the form $G_{n}^{\alpha}$. In this situation, Theorem 2.1 and its proof as mentioned in Remark 2.4 (2) can be applied to obtain both $\|\tau\|$ and $\left\|u^{n / d} \tau\right\|$, provided the geometric action of $\langle u, \tau\rangle$ on $X$, that is, the corresponding smooth epimorphism $\theta: \Lambda \rightarrow\langle u, \tau\rangle$, is known. Indeed, in the proof of Theorem 2.1, we make no use of the fact that $G_{n}^{\alpha}$ is the full automorphism group of $X$ (but just a group acting on it), except when applying Theorem $3.1 \mathrm{in} \mathrm{[4].} \mathrm{This} \mathrm{theorem} \mathrm{is} \mathrm{proved} \mathrm{there} \mathrm{for} \mathrm{the} \mathrm{full} \mathrm{group} \mathrm{Aut}^{ \pm} X$, but the proof actually works for any group containing $\tau$.

It is worth mentioning that the existence of a non-trivial conformal automophism $u$ such that $\tau$ normalizes $\langle u\rangle$ is guaranteed, for example, if the group $\mathrm{Aut}^{+} X$ contains a characteristic abelian subgroup (e.g., if $\mathrm{Aut}^{+} X$ itself is abelian), or if $\mathrm{Aut}^{+} X$ contains an abelian normal Sylow $p$-subgroup (e.g., if $\left|\mathrm{Aut}^{+} X\right|$ is the product of two primes). 


\section{Bounds}

Let $\tau$ be a symmetry on a compact Riemann surface $X$ which admits a nontrivial conformal automorphism $u$ of order $n$ such that $\tau$ normalizes $\langle u\rangle$. In this section we apply Theorem 2.1 to derive upper bounds for the sum of the number of ovals of the conjugacy classes of symmetries of $\langle u, \tau\rangle=G_{n}^{\alpha}$. We also show that the bounds are attained constructing, for infinitely many values of $g, n$ and $\alpha$, a compact Riemann surface of genus $g$ on which $G_{n}^{\alpha}$ acts as a group of automorphisms, and a symmetry $\tau$ in $X$ whose number of ovals reaches the proposed bound. Recall that $d=\operatorname{gcd}(\alpha+1, n)$ and $f=\operatorname{gcd}(\alpha-1, n)$.

We begin with the case $n$ odd.

Proposition 3.1 If $\langle u, \tau\rangle=G_{n}^{\alpha}$ with $n$ odd then

$$
\|\tau\| \leq \frac{g-1}{d}+2
$$

and the bound is attained for every odd $n$ and every $g$ such that $n \mid(g-1)$.

Proof. We first show that the bound is attained provided that $n \mid(g-1)$. In this case, let us consider an NEC group $\Lambda$ with signature $\left(0 ;+;[-] ;\left\{(-)^{k}\right\}\right)$ where $k=(g-1) / n+2$. Let $\theta: \Lambda \rightarrow G_{n}^{\alpha}$ be the homomorphism given by $\theta\left(e_{1}\right)=u^{d}, \theta\left(e_{2}\right)=u^{-d}, \theta\left(e_{i}\right)=1$ for all $i \geq 3, \theta\left(c_{1}\right)=\tau, \theta\left(c_{2}\right)=u^{f} \tau$, $\theta\left(c_{i}\right)=\tau$ for all $i \geq 3$. It is easy to see that it is a well defined smooth epimorphism and so $X=U / \Gamma$, for $\Gamma=\operatorname{ker} \theta$, is a symmetric Riemann surface of genus $g$ on which $G_{n}^{\alpha}$ acts. Corollary 2.3 gives

$$
\|\tau\|=2 \operatorname{gcd}(d, f)+f(k-2)=2+f\left(\frac{g-1}{n}\right)=\frac{g-1}{d}+2 .
$$

To show that this value is an upper bound for $\|\tau\|$, observe that $\tau$ and $u^{n / d} \tau$ generate a dihedral group of order $2 d$ which acts on $X$. Then, Theorem 3(ii) in [2] yields $\|\tau\|+\left\|u^{n / d} \tau\right\| \leq(2 g-2) / d+4$. Since $d$ is odd, both symmetries are conjugate and so $\|\tau\|=\left\|u^{n / d} \tau\right\| \leq(g-1) / d+2$.

Remark 3.2 In the conditions of the above proposition, a genus $g$ Riemann surface $X$ admitting a symmetry with the maximal number $(g-1) / d$ of ovals, can be chosen to have $G_{n}^{\alpha}$ as its full group Aut $^{ \pm} X$ if and only if $n$ is a proper divisor of $g-1$. Indeed, if this is so, then the signature $\sigma=\left(0 ;+;[-] ;\left\{(-)^{k}\right\}\right)$ with $k=(g-1) / n+2 \geq 4$ used in the proof is maximal, and so we may choose a maximal NEC group $\Lambda$ with this signature. With this choice, the surface $U / \Gamma$ constructed there has $G_{n}^{\alpha}$ as its full automorphism group $\mathrm{Aut}^{ \pm} X$. However, if $n=g-1$ then $\sigma$ is not maximal, and 
indeed, an NEC group $\Lambda$ such that $\operatorname{Aut}^{ \pm} X=\Lambda / \Gamma$ cannot have signature $\sigma$; otherwise $\mathrm{Aut}^{+} X$ would act with Fuchsian signature $(2 ;-)$, and it is shown in [1] that in this case $\mathrm{Aut}^{+} X$ would contain a dihedral group. But it is not difficult to see that $\sigma$ is the unique signature providing $(g-1) / d+2=f+2$ ovals. So the number of period cycles of $\Lambda$ is $k \leq 2$ and it can be shown that in this case $\|\tau\| \leq(g-1) / d=f$.

Let us deal now with $n$ even. An upper bound for $\|\tau\|+\left\|u^{n / d} \tau\right\|$ in case $G_{n}^{\alpha}$ is dihedral (i.e., $\alpha=n-1$ ) was given in Lemma 3.3 in [11] and also in Theorems 3(i) and 4(i) in [2]. Namely, $\|\tau\|+\|u \tau\| \leq 4 g / n+2$, and the bound is attained for every $n$ and every $g$ such that $n \mid 4 g$. We now deal with the remainder values of $\alpha$.

Proposition 3.3 Assume that $\langle u, \tau\rangle=G_{n}^{\alpha}$ is not dihedral and $n$ is even.

(1) If $n / f$ is even then $\|\tau\|+\left\|u^{n / d} \tau\right\| \leq(4 g+2 f-n) / d$.

(1.1) If $n /(2 f)$ is even then the bound is attained for every $g, n$ and $\alpha$ such that $n$ is a proper divisor of $4 g+2 f$.

(1.2) If $n /(2 f)$ is odd then the bound is attained for every $g, n$ and $\alpha$ such that $n \mid(2 g+f)$.

(2) Assume $n / f$ is odd.

(2.1) If $n / 2$ is even then $\|\tau\|+\left\|u^{n / d} \tau\right\| \leq(4 g+f-n) / d$, and the bound is attained for every $g, n$ and $\alpha$ such that $(4 g+f) / n$ is an odd integer $\geq 3$.

(2.2) If $n / 2$ is odd then $\|\tau\|+\left\|u^{n / d} \tau\right\| \leq(4 g+4+f-n) / d$, and the bound is attained for every $g, n$ and $\alpha$ such that $n$ is a proper divisor of $4 g+4+f$.

Proof. If $\Lambda$ has signature (1.1) then the Riemann-Hurwitz formula yields $k+\eta h+\sum\left(1-1 / m_{i}\right)+\sum\left(1-1 / n_{i j}\right) / 2=(g-1) / n+2$ where $\eta=1$ or 2 according to the sign of $\Lambda$. In order to maximize the right hand side of formula (2.1) in Theorem 2.1 (keeping constant the area of $\Lambda$ ), it is clear that we first have to deal with NEC groups with $k=1, h=0$ and the maximum number of (even) link periods equal to 2 . Observe that there must exist an elliptic canonical generator $x_{1}$ in $\Lambda$ since the images of the canonical reflections generate a subgroup of $\left\langle u^{n / d}, \tau\right\rangle$, and we are not considering the case $d=n$. So we first have to consider NEC groups $\Lambda$ whose signature has the form

$$
\sigma=\left(0 ;+;[m] ;\left\{\left(2, . v^{\prime} ., 2, d^{\prime}\right)\right\}\right)
$$


with $d^{\prime} \geq 2$ (even) and $m$ as small as possible but such that $\operatorname{lcm}\left(m, d^{\prime}\right)=n$ (to assure the surjectivity of $\theta: \Lambda \rightarrow G_{n}^{\alpha}$ ). Here lcm stands for the least common multiple. Observe that, up to an automorphism in $G_{n}^{\alpha}$, the images of the canonical reflections are of the form $\theta\left(c_{2 i}\right)=\tau, \theta\left(c_{2 i+1}\right)=u^{n / 2} \tau$ for $2 i+1 \leq v^{\prime}$, while $\theta\left(x_{1}\right)=u^{n / m}$. Since $\theta\left(c_{v^{\prime}} c_{v^{\prime}+1}\right)$ has order $d^{\prime}$, we get $\theta\left(c_{v^{\prime}+1}\right)=u^{h n / d^{\prime}} \tau$ where $h$ is prime with $d^{\prime}$ or $d^{\prime} / 2$ according to the parity of $v^{\prime}$. But also $\theta\left(c_{v^{\prime}+1}\right)=\theta\left(e_{1} c_{0} e_{1}^{-1}\right)=u^{(\alpha-1) n / m} \tau$ and so

$$
\operatorname{gcd}\left(h n / d^{\prime}, n\right)=\operatorname{gcd}((\alpha-1) n / m, n) \text {. }
$$

This yields $d^{\prime}=m / \operatorname{gcd}(\alpha-1, m)$ or $d^{\prime}=2 m / \operatorname{gcd}(\alpha-1, m)$. In the first case, $d^{\prime} \mid m$ and so $n=\operatorname{lcm}\left(m, d^{\prime}\right)=m$ and $d^{\prime}=n / f$. In the second, $m=n$ and $d^{\prime}=2 n / f$ if $m$ is even, while $m=n / 2$ and $d^{\prime}=2 n / f$ if $m$ is odd. This leads us to consider the following three signatures:

- $\sigma_{1}=\left(0 ;+;[n] ;\left\{\left(2, . v^{\prime} ., 2, n / f\right)\right\}\right)$ with $v^{\prime}=(4 g+2 f) / n-2$;

- $\sigma_{2}=\left(0 ;+;[n] ;\left\{\left(2, v^{\prime} ., 2,2 n / f\right)\right\}\right)$ with $v^{\prime}=(4 g+f) / n-2$;

- $\sigma_{3}=\left(0 ;+;[n / 2] ;\left\{\left(2, v^{\prime} ., 2,2 n / f\right)\right\}\right)$ with $v^{\prime}=(4 g+f+4) / n-2$ and $n / 2$ odd.

If $n / f$ is even then the largest number of ovals is given by $\sigma_{1}$, namely $\left(v^{\prime}+1\right) n / d=(4 g+2 f-n) / d$. Let us study whether this bound is attained. Observe that $\theta\left(c_{v^{\prime}+1}\right)=u^{\alpha-1} \tau$ and $\theta\left(x_{1}\right)=u$. If $v^{\prime}$ is even then $\theta\left(c_{v^{\prime}}\right)=\tau$ and it is easy to see that $\theta$ is a well defined smooth epimorphism. However, if $v^{\prime}$ is odd, then $\theta\left(c_{v^{\prime}}\right)=u^{n / 2} \tau$, and for $\theta\left(c_{v^{\prime}} c_{v^{\prime}+1}\right)$ to have order $n / f$, it has to be $\operatorname{gcd}(\alpha-1+n / 2, n)=f$; this happens if and only if $n /(2 f)$ is even. Therefore, if $n /(2 f)$ is even then the bound is attained independently of the parity of $v^{\prime}$, while if $n /(2 f)$ is odd, then $v^{\prime}$ is required to be even. This proves part (1) of the proposition.

If $n / f$ is odd and $n / 2$ is even then the largest number of ovals is given by $\sigma_{2}$, namely $(4 g+f-n) / d$. If $v^{\prime}$ is even then $\theta\left(c_{v^{\prime}} c_{v^{\prime}+1}\right)=u^{1-\alpha}$, whose order is not $2 n / f$. Hence there is no smooth epimorphism in this case. However, if $v^{\prime}$ is odd then $\theta\left(c_{v^{\prime}} c_{v^{\prime}+1}\right)=u^{n / 2+1-\alpha}$, which has order $2 n / f$ as is easy to see. So the bound is attained only if $v^{\prime}$ is odd. This proves (2.1).

If $n / f$ and $n / 2$ are odd then the largest number of ovals is given by $\sigma_{3}$, namely $(4 g+4+f-n) / d$. Observe that $v^{\prime}$ is odd. So $\theta\left(c_{v^{\prime}}\right)=u^{n / 2} \tau$ and it is easy to see that $\theta\left(c_{v^{\prime}} c_{v^{\prime}+1}\right)$ has order $2 n / f$. Therefore, $\theta$ is a well defined epimorphism and the bound is attained provided that $v^{\prime}$ is a non-negative integer. This proves (2.2).

Remark 3.4 The symmetries $\tau$ and $u^{n / d} \tau$ generate a dihedral group of order $2 d$, so Theorem 3(i) in [2] yields $\|\tau\|+\left\|u^{n / d} \tau\right\| \leq 4 g / d+2$. This bound is less precise than ours. 


\section{References}

[1] Bujalance, E. And Conder, M. D. E.: On cyclic groups of automorphisms of Riemann surfaces. J. London Math. Soc. (2) 59 (1999), no. 2, $573-584$

[2] Bujalance, E., Costa, A. F. And Singerman, D.: Application of Hoare's theorem to symmetries of Riemann surfaces. Ann. Acad. Sci. Fenn. Ser. A I Math. 18 (1993), no. 2, 307-322.

[3] Bujalance, E., Etayo, J. J., Gamboa, J. M. and Gromadzki, G.: Automorphism Groups of Compact Bordered Klein Surfaces. Lecture Notes in Math. 1439. Springer-Verlag, Berlin, 1990.

[4] Gromadzki, G.: On a Harnack-Natanzon theorem for the family of real forms of Riemann surfaces. J. Pure Appl. Algebra 121 (1997), no. 3, 253269.

[5] Gromadzki, G.: On ovals on Riemann surfaces. Rev. Mat. Iberoamericana 16 (2000), no. 3, 515-527.

[6] Harnack, A.: Über die Vieltheiligkeit der ebenen algebraischen Kurven. Math. Ann. 10 (1876), 189-198.

[7] Klein, F.: Über Realitätsverhältnisse bei der einem beliebigen Geschlechte zugehörigen Normalkurve der $\varphi$. Math. Ann. 42 (1893), no. 1, 1-29.

[8] Izquierdo, M. And Singerman, D.: Pairs of symmetries of Riemann surfaces. Ann. Acad. Sci. Fenn. Math. 23 (1998), no. 1, 3-24.

[9] MelekoĞLu, A.: Symmetries of Riemann Surfaces and Regular Maps. Doctoral thesis, Faculty of Mathematical Studies, University of Southampton, 1998.

[10] Nakamura, G.: The existence of symmetric Riemann surfaces determined by cyclic groups. Nagoya Math. J. 151 (1998), 129-143.

[11] NAtAnzon, S. M.: Finite groups of homeomorphisms of surfaces, and real forms of complex algebraic curves. (Russian). Trudy Moskov. Mat. Obshch. 51 (1988), 3-53, 258. Translation in Trans. Moscow Math. Soc. (1989), $1-51$.

[12] Natanzon, S. M.: On the total number of ovals of real forms of complex algebraic curves. Uspekhi Mat. Nauk (1) 35, 1980, 207-208. (Russian Math. Surveys (1) 35, 1980, 223-224.)

[13] Singerman, D.: On the structure of non-euclidean crystallographic groups. Proc. Cambridge Philos. Soc. 76 (1974), 233-240.

[14] Singerman, D.: Mirrors on Riemann surfaces. In Second International Conference on Algebra (Barnaul, 1991), 411-417. Contemp. Math. 184. Amer. Math. Soc., Providence, RI, 1995. 
[15] Weichold, G.: Über symmetrische Riemannsche Flächen und die Periodizitätsmodulen der zugerhörigen Abelschen Normalintegrale erstes Gattung. Dissertation, Leipzig, 1883.

Recibido: 16 de marzo de 2006

Emilio Bujalance Departamento de Matemáticas Fundamentales Facultad de Ciencias, UNED 28040 Madrid, Spain eb@mat.uned.es

Francisco Javier Cirre Departamento de Matemáticas Fundamentales Facultad de Ciencias, UNED 28040 Madrid, Spain jcirre@mat.uned.es

José Manuel Gamboa Departamento de Álgebra Facultad de Matemáticas, UCM 28040 Madrid, Spain jmgamboa@mat.ucm.es

Grzegorz Gromadzki Institute of Mathematics

University of Gdańsk

Gdańsk, Poland

greggrom@math.univ.gda.pl

E. Bujalance is partially supported by MTM2005-01637. F. J. Cirre is partially supported by MTM2005-01637. J. M. Gamboa is partially supported by MTM2005-20865. G. Gromadzki is supported by the grant SAB2005-0049 of the Spanish Ministry of Education and Sciences and by MTM2005-01637. 